\title{
The Collections Ontology: creating and handling collections in OWL 2 DL frameworks
}

\author{
Paolo Ciccarese, PhD \\ Department of Neurology, Massachusetts General Hospital, Boston, MA, USA \\ Harvard Medical School, Boston, MA, USA. \\ paolo.ciccarese@gmail.com \\ Silvio Peroni, PhD \\ Department of Computer Science and Engineering, University of Bologna, Bologna, Italy. \\ essepuntato@cs.unibo.it
}

Keywords: OWL, collection, list, bag, set

\begin{abstract}
The RDF collections and containers is one of the most used features by RDF technicians and practitioners. However, although some work has been published in past, there is not a standard and accepted way for defining collections within OWL DL frameworks. Trying to address this issue, in this article we introduce the Collections Ontology (CO) version 2.0, an OWL 2 DL ontology developed for creating sets, bags and lists of resources and for inferring collection properties even in presence of incomplete information.
\end{abstract}

\section{Introduction}

In mathematics and computer science, objects that group multiple elements into a single unit, e.g. sets and lists, are commonly known as collections. These entities may involve groups of non-repeatable entities (e.g., students of a particular class), unsorted and repeatable items (e.g., votes of the latest US presidential elections), and even ordered indexes of things (e.g., bibliographic reference lists of scientific papers).

The need of describing those items as belonging to particular collections occurs quite often when formalising real domains through ontologies. Semantic Web technologies (e.g., RDF [1], RDFS [2] and OWL [3]) allow the use of collections to some extent. However, problems arise when we want to define OWL 2 DL ontologies that include known constructs from underlying modelling languages (e.g., RDF sequences and bags).

Several well-known ontologies, e.g. BIBO [4], adopt the aforementioned approach. Of course, such a technique is not an option we want or need to strictly follow the formal constraints given by the OWL 2 DL specifications. In such case, a large amount of ontologies define their own structure for describing collections within OWL 2 DL frameworks. The alternative to this approach is the creation of different and interoperable ways of describing, handling, querying upon entities defined as collections of items.

We envision two possible solutions to properly address collections modelling within OWL 2 DL:

- extend the OWL specification in order to explicitly define mechanisms for handling collections, as happened in RDF, or

- create a standard model for describing collections within OWL 2 DL frameworks, along the line of what has been proposed in [5].

We firmly think that modifications to the OWL specification are not feasible in the short term. For this reason, in this paper we introduce the Collection Ontology $(\mathrm{CO})$, a model for creating collections and align different conceptualisations of them through classes and properties that describe sets, bags and lists of items.
Although this ontology has been first introduced in 2007 as part of the project SWAN (Semantic Web Applications in Neuromedicine) [6], it is been conceived as a separate orthogonal module with no dependencies from the rest of the SWAN ontology ecosystem.

We are here presenting the current version of the $\mathrm{CO}$ ontology (v. 2.0), which has been greatly improved capitalizing on the experience matured in the last four years within several projects and carefully updated to use many of the new features released in OWL 2 DL.

The rest of the paper is structured as follows. In Section 2 we introduce previous approaches to define collections in RDF and OWL. In Section 3 we present the Collections Ontology (CO), describing its main entities and features. Then we show its inference power (Section 4) and how to answer particular queries CO collections through SPARQL (Section 5). In Section 6 we briefly introduce a Java API for creating and handling $\mathrm{CO}$ entities inside a Java application. Finally, we present projects that are making use of $\mathrm{CO}$ for describing different domains (Section 7) and we conclude the paper briefly discussing on future development of our work.

\section{Related Works}

There exists a large amount of literature about Semantic Web models for handling collections of entities. In this section we discuss the most important techniques currently used to address this issue, namely: RDF containers, RDF collections, ontological patterns and OWL ontologies.

\subsection{RDF Containers}

RDF allows the usage of three kinds of containers ${ }^{1}$ :

- rdf:Bag. A bag represents a group of resources or literals, possibly including duplicate members, where there is no significance in the order of the members. For example, a bag might be used to

1 Please note that the current W3C Wroking Draft of RDF 1.1, dated June 5, 2012 (available at http://www.w3.org/TR/rdf11-concepts/), has deprecated such collections without offering an alternative solution. 
describe a group of part numbers in which the order of entry or processing of the part numbers does not matter.

- rdf:Seq. A sequence (or seq) represents a group of resources or literals, possibly including duplicate members, where the order of the members is significant. For example, a sequence might be used to describe a group that must be maintained in alphabetical order.

- rdf:Alt. An alternative (or alt) represents a group of resources or literals that are alternatives (typically for a single value of a property). For example, an alt might be used to describe alternative language translations for the title of a book, or to describe a list of alternative Internet sites at which a resource might be found. An application using a property whose value is an alt container should be aware that it can choose any one of the members of the group as appropriate.

In order to show how to use these constructs, let us take into consideration the following natural language scenario:

The resolution was approved by the Rules Committee, having members Fred, Wilma, and Dino.

We could describe the above scenario in RDF as follows ${ }^{2}$ :

ex:resolution exterms : approvedBy ex:fred, ex:wilma, ex:dino.

However, in the above excerpt we are saying that the resolution is approved by each individual member rather than by the whole group.

Using RDF containers allows us to avoid this issue. In fact, we can use a bag for grouping people as a single unit and then saying that the group approved (property approvedBy) the resolution:

ex:resolution exterms:approvedBy ex:rules-committee . ex:rules-committee a rdf:Bag

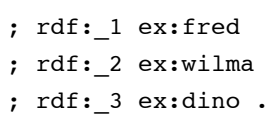

Of course RDF containers have some constraints. In particular, they only state that certain identified resources are members, but they cannot express whether other members that are part of the same container exist. It is not possible to exclude that there might be another graph somewhere that describes additional members.

\subsection{RDF Collection}

RDF provides support for describing groups containing only the specified members, in the form of RDF collections. An $\mathrm{RDF}$ collection is a group of things represented as a list structure (class $r d f:$ List) in the RDF graph.

For instance, we can describe the group of people introduced in the example in the previous section as follows:

ex:resolution exterms:approvedBy ex:rules-committee . ex:rules-committee rdf:first ex:fred

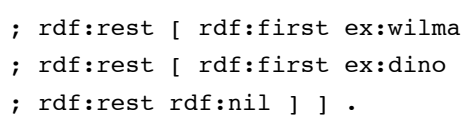

RDF imposes no "well-formedness" conditions on the use of the collection vocabulary - it is possible, for instance, to define multiple $r d f: f i r s t$ elements). Thus, RDF applications

2 The prefixes ex and exterms refer to fictional URLs that describe resources and vocabulary terms respectively. that require collections to be well-formed should be written to check that the collection vocabulary is being used appropriately, in order to be fully robust.

Of course, both RDF/XML and Turtle provide a compact syntaxes for describing collections that avoid the aforementioned "well-formedness" issue, as shown as follows:

ex:resolution exterms : approvedBy

$$
\text { ( ex:fred ex:wilma ex:dino) . }
$$

\subsection{OWL and ordering}

OWL have no support for ordering and the natural constructs from the underlying RDF vocabulary (rdf:List and $r d f: n i l)$ are unavailable in OWL-DL because they are used in its RDF serialization. In principle, $r d f: S e q$ is not illegal but it depends on lexical ordering and has no logical semantics accessible to a DL classifier.

In other terms, as stated in [7]:

- the elements in a container are defined using the relations $r d f: \_1, r d f: \_2$, and so on that have no formal definition in RDF. Using them for the purpose of reasoning will require us to define and enforce the properties of these relations;

- it is not possible to define a container that has elements only of a specific type.

- $\quad$ for updating a specific element in a container in a remote source, one is forced to transmit the whole container.

- it is not possible to associate provenance information with the elements in a container.

Since OWL has greater expressiveness than RDF (with constructs such as transitive properties) and reasoning capabilities (for checking the consistency and inferring subsumptions), the idea of reasoning with sequential structures in OWL-DL looks appealing.

In [5], the authors proposed a way of representing sequential structures in OWL-DL. They argued that the representation of these structures "requires extensive rewriting, the relation of the resulting structures to the original lists is not intuitive and, more importantly, the resulting structures grow as the square of the length of the list". Then, they describe a general list pattern that they incorporated in the Semantic Web Best Practice Working Group's note on n-ary relations [8].

Similar patterns are introduced in [9] and are available as OWL ontologies at the Ontology Design Patterns portal ${ }^{3}$. Among them, the sequence pattern [10] seems to be particularly appropriate for describing sequential structures. In fact, it has been developed primarily for sorting timedependant entities such as tasks, processes, spatially locate objects and situations. Moreover, it defines transitive and intransitive object properties to link an entity of the sequence with its successors and predecessors.

Another not-so-logically-grounded technique for specifying order among entities makes use of literal indexes. The main idea is to aggregate entities in a collection where the order is specified by a value (usually, an integer) defined through data property assertions. For instance, the Music Ontology [11] uses this approach (through the data property track_number) to list the tracks in a record (linked to it with the object property track). Although this approach is very simple, it is very easy to introduce mistakes when modelling such a scenario, for example assigning the same track number to two

3 http://www.ontologydesignpatterns.org 
different tracks of the same record. Usually, this technique prevents common OWL applications from checking automatically the consistency of the ontology unless implementing ad hoc codes.

\section{The Collections Ontology (CO)}

Our contribution in addressing the issue of defining and handling collections within OWL 2 DL frameworks consists in the latest version (2.0) of the Collections Ontology $(\mathrm{CO})^{4}$ or $\mathrm{CO} 2$, originally proposed as part of the SWAN Ontology Ecosystem [6]. As summarised in the Graffoo diagram ${ }^{5}$ in Figure 1, this ontology defines classes and properties that allow one to define three different kinds of collection depending on the particular features that are requested. Namely, sets for describing collections of non-repeatable and unordered elements; bags for defining collections of repeatable and unordered elements; and lists for introducing collections of repeatable and ordered elements. However, before better defining and detailing such classes we would like to explain how $\mathrm{CO}$ relates to the mathematical definition of sets, multisets and sequence.

According to Gregor Cantor, a set is a gathering together into a whole of definite, distinct objects of our perception and of our thought - which are called elements of the set. A set can be described by extension by listing each member of the set. An extensional definition is denoted by enclosing the list of members in curly brackets:

$$
\begin{aligned}
& \mathrm{C}=\{4,2,1,3\} \\
& \mathrm{D}=\{\text { blue, white, red }\}
\end{aligned}
$$

Every element of a set must be unique; no two members may be identical and the order in which the elements of a set or multiset are listed is irrelevant.

A multiset (or bag) is a generalization of the notion of set in which members are allowed to appear more than once. The number of times an element belongs to the multiset is the multiplicity of that member. The total number of elements in a multiset, including repeated memberships, is the cardinality of the multiset. The bag $\{1,2\}$ is also a set.

A sequence is an ordered list of objects (or events). Like a set, it contains members (also called elements or terms), and the number of terms (possibly infinite) is called the length of the sequence. Unlike a set, order matters, and exactly the same elements can appear multiple times at different positions in the sequence.

In general, the identity function operates on the elements of a collection and, if handled, on their order rather then on the collection seen as proper artifact. This means, for instance, that in mathematics two sets containing the same group of elements are the same set, two lists contains the same elements in the same order are the same list, and so on.

In $\mathrm{CO}$ we decided not to model the sets, multisets and sequence in the mathematical sense. We introduced a superclass Collection and to split its subclasses in two disjoint groups according to their ability to consent (i.e., co:Bag and co:List) or not (i.e., co:Set) the repetitiveness of

4 Available at http://purl.org/co.

5 This and all the following graphical representations of ontologies are drawn using Graffoo, the Graphical Framework for OWL Ontologies, available at http://www.essepuntato.it/graffoo. Yellow rectangles represent classes (solid border) and restrictions (dotted border), green parallelograms represent datatypes, arrows starting out of a filled circle refer to object property definitions, arrows starting out of an open circle refer to data property definitions, while other arrows represent assertions between resources. their elements. We therefore defined asserted - manually defined - classes that are not mapping one-to-one to the mathematical classes.

The relationships between the above mathematical entities and those defined by Collections Ontology - and detailed in the following sections of the paper - can be defined as follow:

$$
\begin{aligned}
& \text { co:Set } \sqsubseteq \text { Set } \\
& \text { co:Bag } \subseteq \text { Bag } \\
& \text { co:Set } \sqcap \text { co:Bag }=\varnothing \\
& \text { co:List }=\text { co:Bag } \sqcap \text { Sequence }
\end{aligned}
$$

There are precise pragmatic reasons motivating this design choice. First of all, we chose not to model the mathematical identity function in $\mathrm{CO}$ for a specific reason: to allow one to use $\mathrm{CO}$ even when modelling scenarios that describe "collections in terms of the constructive boundaries of those plural entities that form themselves a whole" [13]. Therefore, it is possible to consider two sets of people, composed exactly by the same people, as two different research groups without contradictions. A more extensive example of this use is shown in section 4 , in which we introduce how to use this feature to leverage inference. Second, from an implementation standpoint, the data structures managing co:Set and co:Bag are very different. Last but not least, we decided to design the new version of $\mathrm{CO}$ as back compatible with its previous versions, since they are currently used in implemented systems and frameworks, as we introduce in Section 7.

\section{[INSERT Figure 1 HERE]}

The main improvements introduced in this version of $\mathrm{CO}$ are:

- All the entities are assigned to a new URL base, i.e. "http://purl.org/co/";

- The existing logical structure of the ontology has been partially re-organised;

- Addition of new properties describing inverse relations and indexes of list items;

- Use of new OWL 2 DL capabilities to offer a better inference layer;

- Introduction of additional logical axioms and SWRL rules for improved consistency checking and integrity constraints;

- Addition of natural language labels and comments for improving the human-understanding of $\mathrm{CO}$;

- An accompanying ontology ${ }^{6}$ that aligns the current version of $\mathrm{CO}$ with the old version developed for SWAN and with other ontologies handling collections;

- A Java API so as to load, manage and store CO collections within a Java application;

In the following subsections, we introduce all the main classes and properties defined in $\mathrm{CO}$, supporting them through exemplar use cases.

\subsection{Collection}

The class co:Collection is the top-level "abstract" class of $\mathrm{CO}$. Any individual of this class can only contain elements as OWL entities (i.e., individuals of the class owl:Thing) and must specifies a particular size (property co:size). It is the

6 http://purl.org/co/alignment 
superclass of the "concrete" collections of CO, i.e., co:Set, co:Bag and co:List - we introduce in the following sections. This class and its related properties are defined as follows:

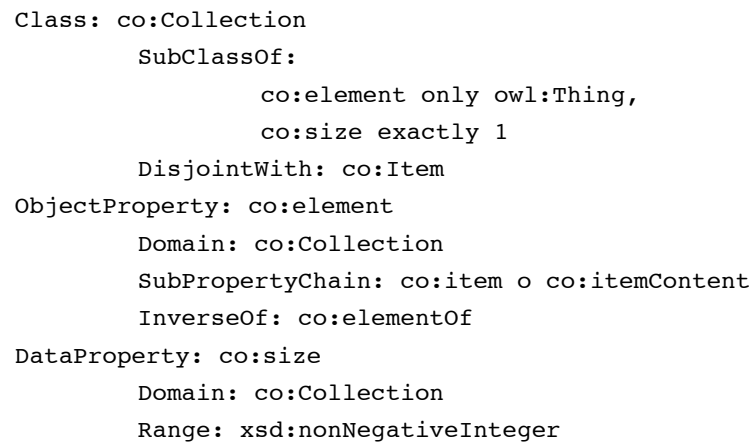

Note that the size of a collection $C$ refers to the number of times $\mathrm{C}$ refers to its elements. For example, the following collections - composed (property co:element) by the same three elements $a, b$ and $c$-have all different sizes:

- the size of the set $\{a, b, c\}$ is 3 ;

- the size of the bag $[a, b, b, c, a]$ is 5 ;

- $\quad$ the size of the list $(a, b, c, a, a, c, b)$ is 7 .

\subsection{Set}

An individual of the class co:Set is a collection that cannot contain duplicate elements. All the elements of the set are directly linked to it through the property co:element, as shown in Figure 2. This class is defined as follows7:

Class: co: Set

Subclassof: co:Collection

\section{[INSERT Figure 2 HERE]}

In OWL, identical elements connected by the same property are, by default, treated as items of a set.

Let us take again into consideration the example introduced in Section 2.1. Using CO sets, it is possible to describe easily that scenario as follows:

ex:resolution exterms:approvedBy ex:rules-committee . ex:rules-committee a co:set

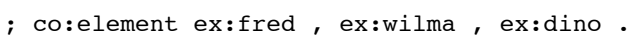

\subsection{Bag}

An individual of the class co:Bag (that is disjoint with $c o:$ Set) is a collection that can have multiple copies of each element. As shown in Figure 3, this is performed through the class co:Item and the property co:item. The class co:Item links exactly one resource the effectively is contained in the bag through the relationship co:itemContent. The dereferencing mechanism implemented through the properties co:item and co:itemContent allows, then, to associate a same resource to a collection more than one time. This class and its related properties are defined as follows:

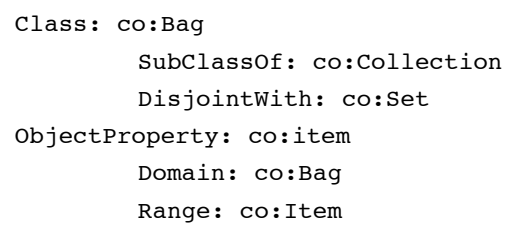

7 This and all the following excerpts of ontology models are written according to the Manchester Syntax, while all the examples of use of the model are written in Turtle.

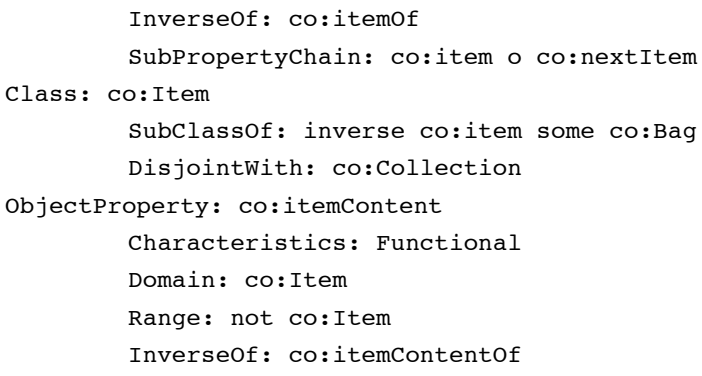

[INSERT Figure 3 HERE]

Bags can be used in all those scenarios where we do not care about the order and we want to keep track of repeatability of elements. The following example introduces a simple context in which bags can be used for:

\section{The factorisation of the number 20 is " $2,2,5$ ".}

Since the order of the prime factors in the factorisation is not important for mathematical purposes, we can use $\mathrm{CO}$ bags to describe the above scenario in OWL:

ex: twenty exterm:hasfactorisation ex:twenty-factors . ex: twenty-factors a co:Bag ; co:item ex:i1, ex:i2, ex:i3.

ex:il a co:Item; co:itemContent ex:two .

ex:i2 a co:Item; co:itemcontent ex: two.

ex:i3 a co:Item; co:itemContent ex:five.

Moreover, by means of the OWL 2 feature for defining property chains, it has been possible to infer automatically the belongingness to a bag, i.e., all the co:element relations between a bag instance and all the other objects it effectively contains, that are dereferenced through items and the related properties co:item and co:itemContent for allowing repetition.

\subsection{List}

An individual of the class co:List (that is subclass of co:Bag) is an abstract data structure that implements an ordered collection of elements, where the same element may occur more than once. As shown in Figure 4, the ordering is performed through the property co:nextItem that links an individual of the class co:ListItem (subclass of co:Item) to exactly another one. Moreover, co:nextItem is accompanied by its related inverse and transitive properties. As for co:Item, the class co:ListItem links exactly one resource through the relationship co:itemContent.

\section{[INSERT Figure 4 HERE]}

In order to identify which are the first and the last items in a list, two object properties are defined, co:firstItem and co:lastItem, as sub-property of co:item. Of course, list items linked through these two properties cannot be respectively preceded or followed by another list item. This class and its related properties are defined as follows:

Class: co:List

Subclassof :

co:firstItem exactly 1 ,

co:lastitem exactly 1 ,

co:Bag that co:item only co:ListItem

ObjectProperty: co:firstitem

Characteristics: Functional

SubPropertyof: co:item

Domain: co:List 
Range: co:ListItem that

co:previousitem exactly 0 and

co: index value 1

Inverseof: co:firstItemof

ObjectProperty: co:lastItem

Characteristics: Functional

SubPropertyof: co:item

Domain: co:List

Range: co:ListItem that

co: nextItem exactly 0

InverseOf: co:lastItemof

Class: co:ListItem

Subclassof: co:Item that co:index exactly 1

ObjectProperty: co:followedBy

Characterstics: Transitive

Domain: co:ListItem

Range: co:ListItem

ObjectProperty: co:precededBy

Characteristics: Transitive

InverseOf: co:followedBy

ObjectProperty: co:nextItem

Characteristics: Functional

SubPropertyof: co:followedBy

ObjectProperty: co:previousItem

Characteristics: Functional

SubPropertyof: co:precededBy

Inverseof: co:nextItem

DataProperty: co:index

Domain: co:ListItem

Range: xsd:positiveInteger

Let us introduce an example to show how to use CO lists for describing ordered collections. Suppose one wants to describe the paper referenced by [5] specifying its authors (e.g., through the property dcterms:creator) in that specific order. It is possible to model this scenario straightforwardly using a CO list as follows ${ }^{8}$ :

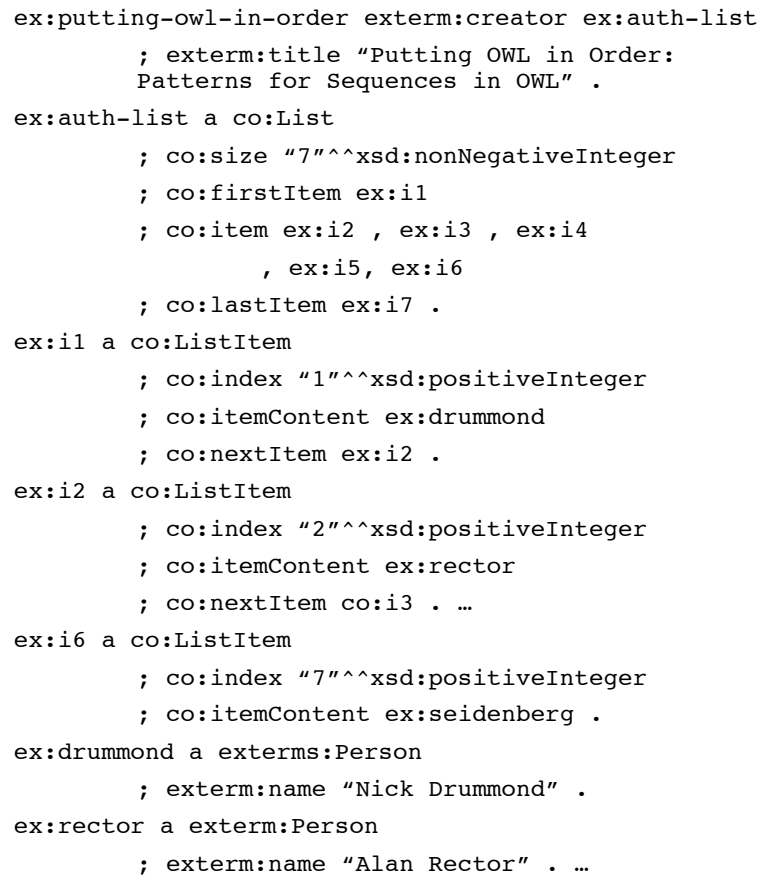

Following this methodology, it is possible to keep separate the elements involved in a list (i.e., the authors of the paper in

8 The prefixes $x s d$ and dcterms in the following examples refer to the XML Schema (http://www.w3.org/2001/XMLSchema\#) and the DCTerms (http://purl.org/dc/terms/) vocabularies respectively. the previous example) and the position that those elements occupy in a particular list. This feature is particularly important when the same element can be part (at different indexes) of more then one list (e.g., a person can be first author of a paper and third author of another.

\subsubsection{Leave it to the inference layer}

In $\mathrm{CO}$, the lists are defined in a way that is possible to consider some data as implicit, leaving to a reasoner or an inference system the job of inferring them.

For example, it is not needed to explicitly specify all the items that are involved in a list. In fact, through the following property chain axiom defined for the property co:item:

co:item o co:nextItem

it is possible not to specify all the items of a list, but just the first (property co:firstItem) and the last (property co:lastItem) ones. In this way, the reasoner will be able to infer all the remaining co:item assertions simply following the chain of co:nextItem defined by the list items.

Moreover, the combination of the above property chain can be very useful when combined with the following SWRL rules [12]:

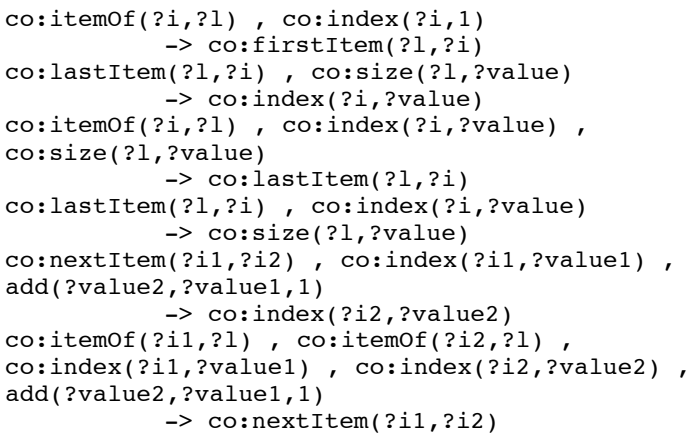

Through this inference layer, it is then possible to complete lists even when they present partial information, in particular identifying:

- the first item of a list starting from its index;

- the last item of a list starting from its index and the related list size (and vice versa);

- the size of the list from its last item;

- indexes of items starting from their co:nextItem assertions (and vice versa)

\subsubsection{Integrity constraints}

The transitive properties co:followedBy and co:precededBy (super-properties of co:nextItem and co:previousItem respectively) are used to indicate all the items that follows/precedes a particular item. In $\mathrm{CO}$, no cycles are permitted, i.e., an item cannot either follow or precede itself. OWL 2 allows one to set this behaviour for object properties specifying them as irreflexive. However, it is not possible to set those two properties as irreflexive since it would violate one of the needed keep the ontology in a DL framework ${ }^{9}$.

Since the constraint on co:followedBy and co:precededBy is fundamental to keep the ontology consistent, we chose to specify integrity constraints by means of a particular model: the Error Ontology ${ }^{10}$. This ontology is a unit test that allows producing an inconsistent model if a particular (and incorrect) situation happens. It works by means of a data

9 In this particular case, it is not possible to specify an object property as transitive and irreflexive at the same time.

10 Available at: http://www.essepuntato.it/2009/10/error. The prefix error refers to entities defined in it. 
property, error:hasError, that denies its usage for any resource, as shown as follows:

DataProperty: error:hasError

Domain: error:hasError exactly 0

Range: xsd:string

In fact, by defining its domain as "all those resources that do not have any error:hasError assertion", a resource that asserts having an error makes automatically the ontology inconsistent $^{11}$.

By means of the Error Ontology, we can mandate the properties co:followedBy and co:precededBy to be, implicitly, irreflexive. This behaviour is implemented through the following SWRL rules ${ }^{12}$ :

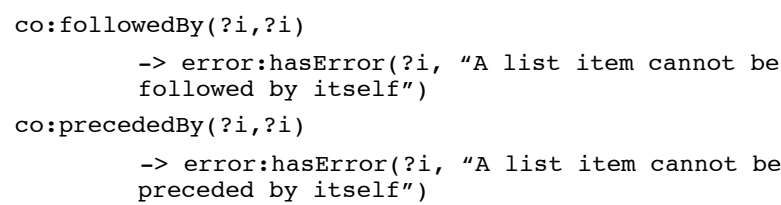

\section{Leveraging inference}

The Open Reuse and Exchange specification (ORE specification) [14] is a standard defined by the Open Archives Initiative for describing and exchanging aggregations of Web resources.

The main concept of this specification is the Aggregation, i.e., a particular resource that aggregates, either logically or physically, other resources. It is also possible to use particular kinds of resources called proxies, so as to refer to a specific aggregated resource in a context of a particular aggregation. Moreover, by using proxies, we can specify an order (with an external vocabulary) for aggregated resources of an aggregation, if needed.

Let us briefly introduce the use of ORE for a real-world scenario. For instance our personal scientific library, composed by a large number of works, can be seen as an aggregation of different papers. We can use ORE to describe this scenario ${ }^{13}$ :

$$
\begin{aligned}
& \text { ex:my-own-library a ore:Aggregation } \\
& \text {; ore:aggregates } \\
& \text { ex:putting-owl-in-order } \\
& \text {, w3:rdf-concepts } \\
& \text {, w3 }: \text { rdf-sparql-query . }
\end{aligned}
$$

Another exemplar aggregation in the same context can be the bibliographic reference list of a particular article. When we are writing a scientific paper, we use to refer to bibliographic references, each of them referencing a precise paper, for explicitly citing other works in our paper. Of course, two bibliographic references, even when defined in two different

11 Of course, the Collection Ontology could be forced to be inconsistent in a simpler way that doesn't require the use of the property error:hasError - e.g specifying a rule such as followedBy(?i,?i) -> owl:Nothing(?i). However, we prefer to specify an error message, which can be very useful when used with automated debugging tools.

12 It is important to notice that all these rules do not work at the Tbox level and, thus, you need an Abox to be correctly applied. In addition, they also do not work with anonymous individuals since the DL safe rules contraint must hold to use SWRL rules within OWL ontologies. We are aware of of this constraint and, even though all the examples in the previous sections make use of several black nodes (i.e. anonymous individuals) thus making these SWRL rules unusable, we decided to use such black nodes for the sake of clarity.

13 The prefixes ore and $w 3$ refer respectively to http://www.openarchives.org/ore/items/ and http://www.w3.org/TR/ papers and referring to the same work, can have associated particular (and contextual) metadata that change reference by reference. This scenario can be described in OWL through ORE as follows:

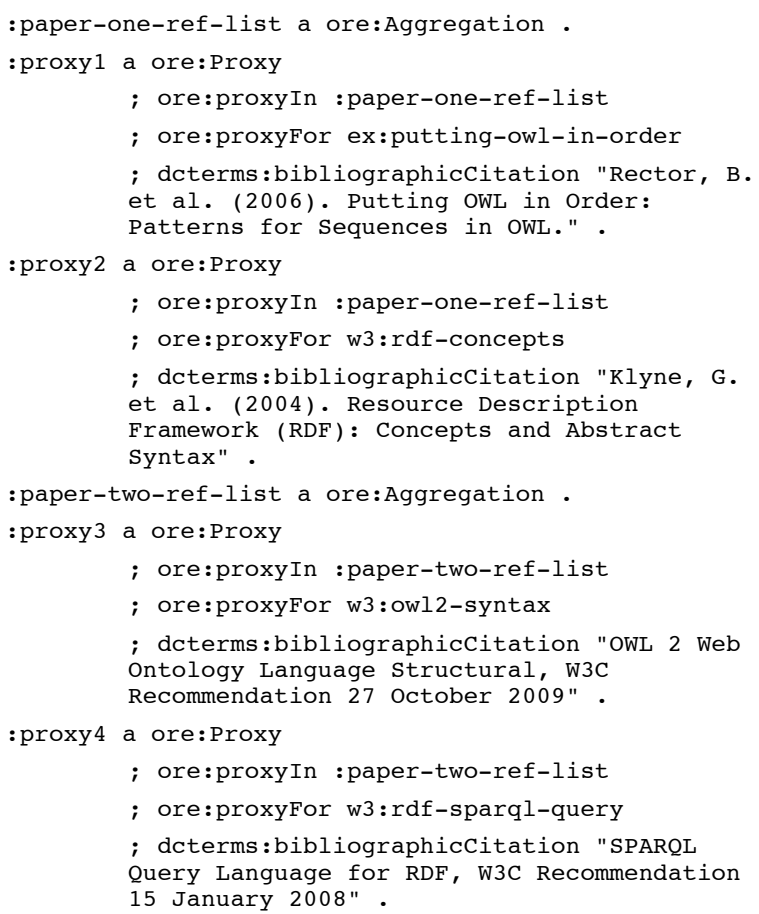

ORE does not require to use a specific vocabulary for describing the order between proxies. Since the order in a reference list is usually important to handle, we can use $\mathrm{CO}$ with ORE in order to describe proxies sorting, adding the following statements:

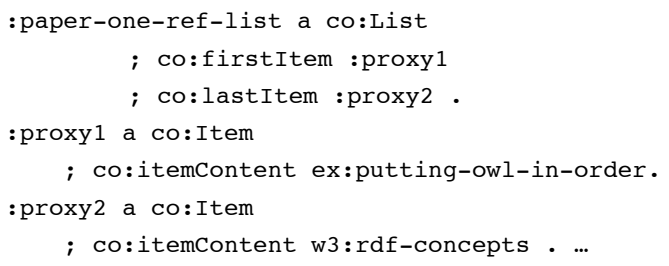

Of course, ore:Aggregation and co:List are used in a very redundant way in the above excerpts. Adding an additional layer of ontological alignment between the two ontologies can help in obtaining the same set of data writing just some of them. For instance, we can add the following (Manchester Syntax) axioms to ORE with the explicit goal of leveraging inference:

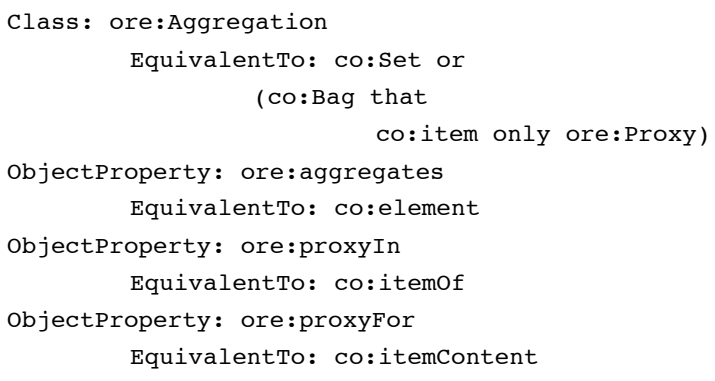

In this way, it becomes possible to re-write a less verbose definition of the first reference list of the above examples as follows:

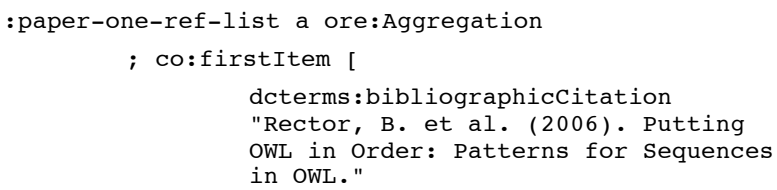


; co:nextItem [

dcterms:bibliographicCitation

"Klyne, G. et al. (2004). Resource

Description Framework (RDF):

Concepts and Abstract Syntax" ] ] .

\section{Querying CO datasets}

$\mathrm{CO}$ allows one to make very sophisticated SPARQL queries [15] to datasets containing information structured as $\mathrm{CO}$ collections. In this section we introduce just few query samples, of incremental complexity, in order to highlight how $\mathrm{CO}$ is able to treat even complicated scenarios. In the next examples, we take into consideration the data described in Section 3.3.

Query: "Give me all the author collections containing persons named "Alan Rector"'.

SELECT DISTINCT ?collection

WHERE \{ ?paper exterm:creator ?collection .

?collection co:element [ a exterm:Person

; exterm:name "Alan Rector" ] \}

Query: "Give me all the papers written by persons named 'Alan Rector' and not 'Nick Drummond", 14 .

SELECT DISTINCT ?paper

WHERE \{ ?paper exterm:creator ?collection . ?collection co:element [ a exterm:Person ; exterm:name "Alan Rector" ]

FILTER NOT EXIST \{ ?collection co:element [

$$
\text { a exterm: Person }
$$$$
\text { ; exterm:name "Nick Drummond" \} \} }
$$

Query: "Tell me how many author lists contain persons named 'Alan Rector"'.

SELECT (COUNT(DISTINCT ?item) AS ?number)

WHERE $\{$ ?paper exterm:creator [

?item a co:ListItem

; co:itemContent [ a exterm:Person

; exterm:name "Alan Rector" ] ] \}

Query: "Give me all the author lists where persons are named 'Alan Rector' are either first or second author".

SELECT DISTINCT ?list

WHERE \{ ?paper exterm:creator ?list .

?author a exterms:Person

exterms:name "Alan Rector".

?list co:firstItem ?first .

\{ ?first co:itemContent ?author \}

UNION

$\{$ ?first co:nextItem [

co:itemContent ?author ] \} \}

Query: "Give me all the papers and their respective authors ordered by their positions."

SELECT DISTINCT ?paper ?person

WHERE $\{$ ?paper exterm:title ?title

; exterm:creator [ co:item [

co:index ?position

co:itemContent ?author ] ]

\} ORDER BY ?title ?position

14 In the following SPARQL query we use the construct "FILTER NOT EXISTS" to get out the correct answer. This approach only works because of the SPARQL processor evaluates the query accordin to a close-world point of view, contrarily to what is prescribed by OWL ontologies in general, that strictly follow the open-world assumption. Thus, it is important to clarify there is nothing in the Collection Ontology that allows a reasoner to prove a list do not contain a particular.

\section{A Java API}

Even when an ontology is well-developed and useful to describe a particular domain, it still remains just a theoretical model if it is not accompanied by an API that allows one to use the model inside software applications. To this end, we developed a complete and extensible Java API for $\mathrm{CO}^{15}$. It allows one to create/modify and load/store CO entities directly from a Java code. It is composed by a base package (i.e., "org.purl.co") that implements the core classes for handling CO collections in Java. Moreover, it includes general interfaces for loading/storing an environment of $\mathrm{CO}$ collections from/into files or input/output streams.

Our API is a general-purpose library that is easy to be integrated with any other RDF/OWL APIs such as Jena [16] and OWLAPI [17]. This is possible by implementing the interfaces COReader and COWriter so as to have mechanism to handle RDF resources through the favourite Java library.

In the following excerpts, we introduce the use of our own Jena extension to the CO API. The first thing to do is to create a new $\mathrm{CO}$ environment (interface COEnvironment) in which we can handle collection of Jena resources (interface Resource):

COEnvironment $<$ Resource $>$ env $=$

new StandardcoEnvironment<Resource $>$ ( ) ;

Each $\mathrm{CO}$ environment makes available all the methods for creating new CO collections, i.e. sets (method createCOSet, that returns a COSet object), bags (method createCOBag, that returns a COBag object) and lists (method createCOList, that returns a COList object). Through these interfaces and methods, the creation of the list introduced in Section 3.3 becomes straightforward:

Model $\mathrm{m}=$ ModelFactory.createDefaultModel ();

String ex = "http://www.example.com/ex/";

COList<Resource $>$ auth-list $=$

env.createCOList(

$$
\text { URI.create (ex+"auth-list")); }
$$

auth-list.add (m.createResource (ex+"drummond")); auth-list.add(m.createResource(ex+"rector")) ; ... auth-list.add (m.createResource (ex+"seidenberg") );

Finally, it is possible to load/store an environment from/into files or other input/output streams through implementation of the interfaces COReader and COWriter. For instance, to store the previous defined list in a particular file using the Turtle format we need to create a new writer, specifying the destination format, and then store the environment in a file:

Cowriter $<$ Resource $>$ writer $=$

$$
\text { new JenaRDFWriter(Format.Turtle); }
$$

File destination = new File ("mylist.ttl");

writer.store (env, destination);

Beside these basic operations, the API implements Java methods and classes for all the OWL properties and classes defined in CO. Moreover, it includes mechanisms to guarantee the correctness and consistency of all the collections one creates.

\section{Who is using CO}

The Collections Ontology has been already adopted by the Semantic Web applications and projects introduced in this section.

$15 \quad$ Available at: http://code.google.com/p/collections-
ontology/downloads




\subsection{SWAN}

The SWAN project ${ }^{16}$ (Semantic Web Applications in Neuromedicine) aims to develop a practical, common, semantically structured framework for biomedical discourse initially applied, but not limited, to significant problems in Alzheimer Disease (AD) research. AlzSWAN ${ }^{17}$ an AD knowledge base created in collaboration with the Alzheimer Research Forum ${ }^{18}$ represents the most popular instance of the SWAN platform. It consists in a network of about 2400 research statements linked to about 2700 publications.

The SWAN biomedical discourse ontology [6] represents the backbone of the project and its purpose is to function as the schema of a distributed knowledgebase in $\mathrm{AD}$ and to link information in that knowledgebase with other information in biomedicine. Back in 2007, the SWAN ontology has been architected as a set of orthogonal modules that combines into the SWAN ontology ecosystem.

One of such modules was the first version of Collections Ontology as collections are necessary to manage several aspects of the scientific discourse modeling. For example, a scientific argument can be represented by a sequence of research statements such as hypothesis, claims and questions. And their order is crucial as a way to convey the hypothesis properly.

The SWAN platform features have been incrementally embedded in the new Domeo Annotation Toolkit ${ }^{19}$ [18] an extensible web application enabling users to visually and efficiently create and share ontology-based stand-off annotation on HTML or XML document targets. Domeo supports manual, fully automated, and semi-automated annotation with complete provenance records, as well as personal or community annotation with access authorization and control. Domeo uses the SWAN ontology and Collections Ontology for representing scientific discourse.

\subsection{EARMARK}

The Extremely Annotational RDF Markup (EARMARK) [19$20]$ is a new markup meta-language defined by means of Semantic Web technologies. The basic idea is to model EARMARK documents as collections of addressable text fragments, and to associate such text content with OWL assertions that describe structural features as well as semantic properties of (parts of) that content. As a result EARMARK allows not only documents with single hierarchies (as with XML) but also multiple overlapping hierarchies where the textual content within the markup items belongs to some hierarchies but not to others. Moreover EAMARK makes it possible to add semantic annotations to the content though assertions that may overlap with existing ones.

EARMARK is defined by an OWL ontology ${ }^{20}$ that models all the classes and properties for describing typical markup structures, such as elements, attributes, text nodes, parentchild relations and the like. From an ontological perspective, EARMARK documents are just ABox of cited ontology.

One of the most important features that must be supported in document markup languages is the possibility of specifying a particular order between items (e.g., elements and attributes). The EARMARK ontology implements this feature importing

16 Available at: http://swan.mindinformatics.org

17 Available at: http://hypothesis.alzforum.org

18 Available at: http://alzforum.org

19 Available at: http://annotationframework.org

20 Available at: http://www.essepuntato.it/2008/12/earmark the (old version) of $\mathrm{CO}$. This makes it possible to handle markup items as collections of other ordered or unordered, repeatable or non-repeatable items.

A new version of EARMARK (both the ontology and its Java $\mathrm{API}^{21}$ ) is now in-development with the aim of adopting the current version of $\mathrm{CO}$, so as to take advantage from all its new features and inferential power.

\subsection{SPAR}

The Semantic Publishing and Referencing Ontologies $(S P A R)^{22}$ is a suite of orthogonal and complementary OWL 2 DL ontology modules. They together permit the creation of comprehensive machine-readable RDF metadata for all aspects of semantic publishing and referencing: documents description, types of citations and their related contexts, bibliographic references, document parts and status, agents' roles and workflow processes, etc.

Some of the SPAR ontologies, such as the FRBR-aligned Bibliographic Ontology (FaBiO) [21], suggest explicitly to use $\mathrm{CO}$ for handling scenarios in which specifying an order among entities is mandatory (e.g., the list of the authors of a paper). Others, such as the Bibliographic Reference Ontology $(B i R O)$, import directly $\mathrm{CO}$ for handling particular purposes, such as describing reference lists in research articles.

\section{Conclusions}

One of the most important and used features of existing RDF data is the possibility of defining collections and containers to group resources as one entity. This characteristic has not been included in OWL since its beginning, even in its latest OWL 2 DL specification. Alternative proposals has been done in past for addressing this issue, but it seems they do not come to develop a shared standard for defining collections within OWL DL frameworks.

In this paper we introduced the Collections Ontology $(\mathrm{CO})$ version 2.0, our OWL 2 DL ontology developed specifically for addressing the issue of defining collection in OWL frameworks. In particular, we introduced the graphical and formal description of the ontology and we provided examples of usage in terms of Abox modelling, inferences and SPARQL queries. In addition to what we illustrated here, more information and examples are also available in the project website ${ }^{23}$.

One of the most immediate future developments for our work is the extension of the Java API so as to release libraries to be used with other Java OWL environments, such as OWLAPI, as well as the porting of the current API in different program languages.

\section{Acknowledgements}

We thank Jonathan Rees for his valuable feedback, Tim Clark for his support and finally to some anonymous experts who gave us several suggestions in terms of terminology, modelling and presentation.

\section{References}

\footnotetext{
21 Available at: http://palindrom.es/phd/research/earmark 22 Available at: http://purl.org/spar 23 Collections Ontology (CO) Google Code project available at: http://code.google.com/p/collections-ontology
} 
1. Carroll, J., Klyne, G. (2004). Resource Description Framework (RDF): Concepts and Abstract Syntax. W3C Recommendation, 10 February 2004. World Wide Web Consortium. http://www.w3.org/TR/rdf-concepts/ (last visited December 27, 2012).

2. Brickley, D., Guha, R.V. (2004). RDF Vocabulary Description Language 1.0: RDF Schema. W3C Recommendation, 10 February 2004. World Wide Web Consortium. http://www.w3.org/TR/rdf-schema/ (last visited December 27, 2012).

3. Motik, B., Patel-Schneider, P. F., Parsia, B. (2009). OWL 2 Web Ontology Language: Structural Specification and Functional-Style Syntax. W3C Recommendation, 27 October 2009. World Wide Web Consortium. http://www.w3.org/TR/owl2-syntax/ (last visited December 27, 2012).

4. D'Arcus, B., Giasson, F. (2009). Bibliographic Ontology Specification. Specification Document, 4 November 2009. http://bibliontology.com/specification (last visited December 27, 2012).

5. Drummond, N., Rector, A., Stevens, R., Moulton, G., Horridge, M., Wang, H. H., Seidenberg, J. (2006). Putting OWL in Order: Patterns for Sequences in OWL. In Grau, B. C., Hitzler, P, Shankey, C., Wallace, E. (Eds.), Proceedings of the Workshop on OWL: Experiences and Directions (OWLED 2006). Aachen, Germany: Sun SITE Central Europe. http://sunsite.informatik.rwthaachen.de/Publications/CEUR-WS/Vol216/submission_9.pdf (last visited December 27, 2012).

6. Ciccarese, P., Wu, E., Kinoshita, J., Wong, G., Ocana, M., Ruttenberg, A., Clark, T. (2008). The SWAN Biomedical Discourse Ontology. Journal of Biomedical Informatics, $41 \quad$ (5), 739-751. DOI: 10.1016/j.jbi.2008.04.010.

7. Chaudhri, V. K., Jarrold, B., Pacheco, J. (2006). Exporting Knowledge Bases into OWL. In Grau, B. C., Hitzler, P, Shankey, C., Wallace, E. (Eds.), Proceedings of the Workshop on OWL: Experiences and Directions (OWLED 2006). Aachen, Germany: Sun SITE Central Europe. http://sunsite.informatik.rwth-aachen.de/ Publications/CEUR-WS/Vol-216/submission_34.pdf (last visited December 27, 2012)

8. Hayes, P., Welty, C. (2006). Defining N-ary Relations on the Semantic Web. W3C Working Group Note, 12 April 2006. World Wide Web Consortium. http://www.w3.org/TR/swbp-n-aryRelations/ (last visited December 27, 2012).

9. Presutti, V., Gangemi, A. (2008). Content Ontology Design Patterns as practical building blocks for web ontologies. In Li, Q., Spaccapietra, S., Yu, E. S. K., Olivé, A. (Eds.), Proceedings of the 27th International Conference on Conceptual Modeling (ER 2008). Heidelberg, Germany: Springer.

10. Gangemi, A. (2010). Submission: Sequence. http://ontologydesignpatterns.org/wiki/Submissions:Sequ ence (last visited December 27, 2012).

11. Raimond, Y., Giasson, F. (2010). Music Ontology Specification. Specification Document, 28 November 2010. http://musicontology.com/ (last visited December 27, 2012).

12. Horrocks, I., Patel-Schneider, P. F., Boley, H. Tabet, S., Grosof, B., Dean, M. (2004). SWRL: A Semantic Web Rule Language Combining OWL and RuleML. W3C Member Submission 21 May 2004. World Wide Web
Consortium. http://www.w3.org/Submission/SWRL/ (last visited December 27, 2012).

13. Bottazzi, E., Catenacci, C., Gangemi, A., Lehmann, J. (2006). From collective intentionality to intentional collectives: An ontological perspective. In Cognitive Systems Research, 7 (2\&3): 192-208. DOI: 10.1016/j.cogsys.2005.11.009.

14. Lagoze, C., Van de Sompel, H., Johnston, P., Nelson, M., Sanderson, R., Warner, S. (2008). Abstract Data Model. Object Reuse and Exchange Specification. Open Archives Initiative. http://www.openarchives.org/ ore/1.0/datamodel (last visited December 27, 2012).

15. Harris, S., Seaborne, A. (2011). SPARQL 1.1 Query Language. W3C Proposed Recommendation, 08 November 2012. World Wide Web Consortium. http://www.w3.org/TR/sparq111-query/ (last visited December 27, 2012).

16. Carroll, J., Dickinson, I., Dollin, C., Reynolds, D., Seaborne, A., Wilkinson, K. (2004). Jena: implementing the semantic web recommendations. In Feldman, S. I., Uretsky, M., Najork, M., Wills, C. E. (Eds.), Proceedings of the 13th international conference on World Wide Web - Alternate Track Papers \& Posters (WWW 2004). New York, New York, USA: ACM.

17. Horridge, M., Bechhofer, S. (2011). The OWL API: A Java API for OWL ontologies. In Semantic Web Interoperability, Usability, Applicability, 2 (1): 11-21. DOI: 10.3233/SW-2011-0025.

18. Ciccarese, P., Ocana, M., Clark, T. (2011). DOMEO: a web-based tool for semantic annotation of online documents. Paper at Bio-Ontologies 2011, Vienna, Austria.

19. Di Iorio, A., Peroni, S., Vitali, F. (2011). A Semantic Web Approach To Everyday Overlapping Markup. In Journal of the American Society for Information Science and Technology, 62 (9): 1696-1716. DOI: 10.1002/asi.21591.

20. Di Iorio, A., Peroni, S., Vitali, F. (2011). Using Semantic Web technologies for analysis and validation of structural markup. In International Journal of Web Engineering and Technologies, 6 (4): $375-398$. DOI: 10.1504/IJWET.2011.043439

21. Peroni, S., Shotton, D. (2012). FaBiO and CiTO: ontologies for describing bibliographic resources and citations. In Journal of Web Semantics: Science, Services and Agents on the World Wide Web, 17 (December 2012): 33-43. DOI: 10.1016/j.websem.2012.08.001 


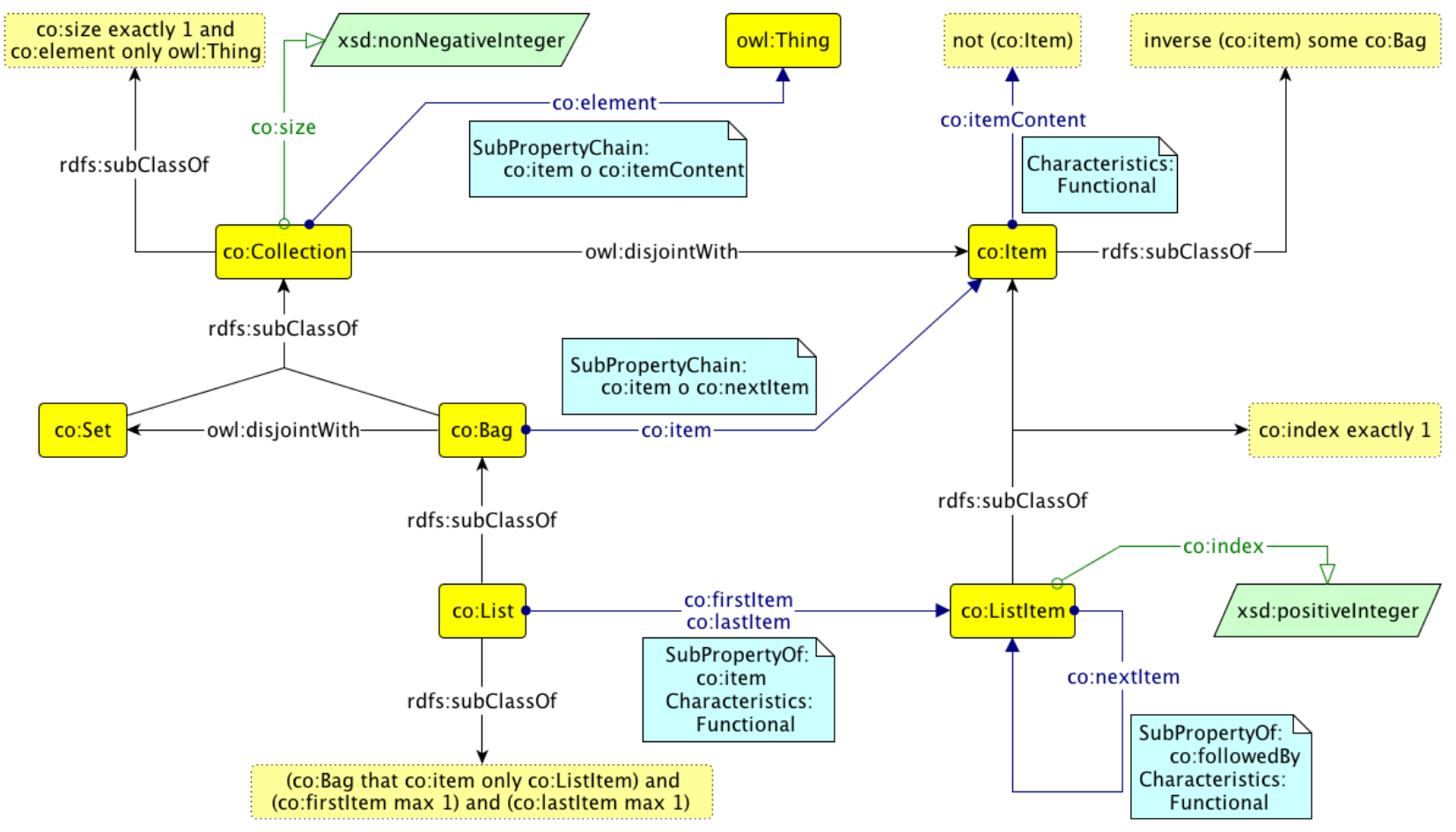

Figure 1. Diagram summarising the main structure of the Collections Ontology. 


$$
\text { owl:Thing }
$$

Figure 2. Diagram summarising the class Set and the related property element. 


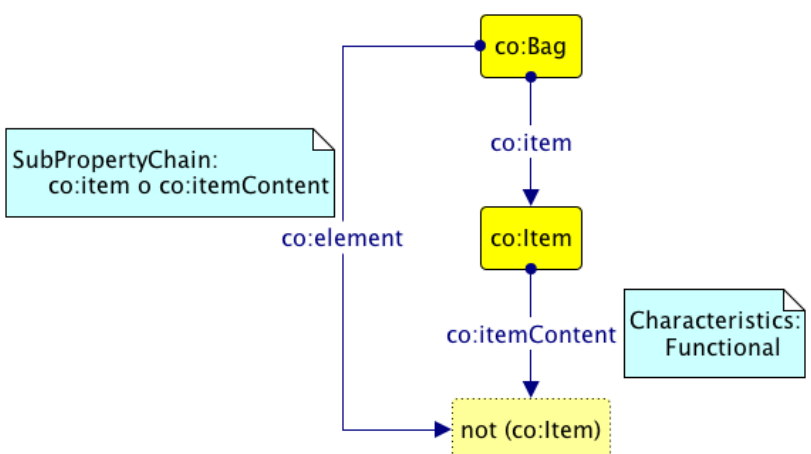

Figure 3. Diagram summarising the class Bag and the related class Item and properties item, itemContent and element. 


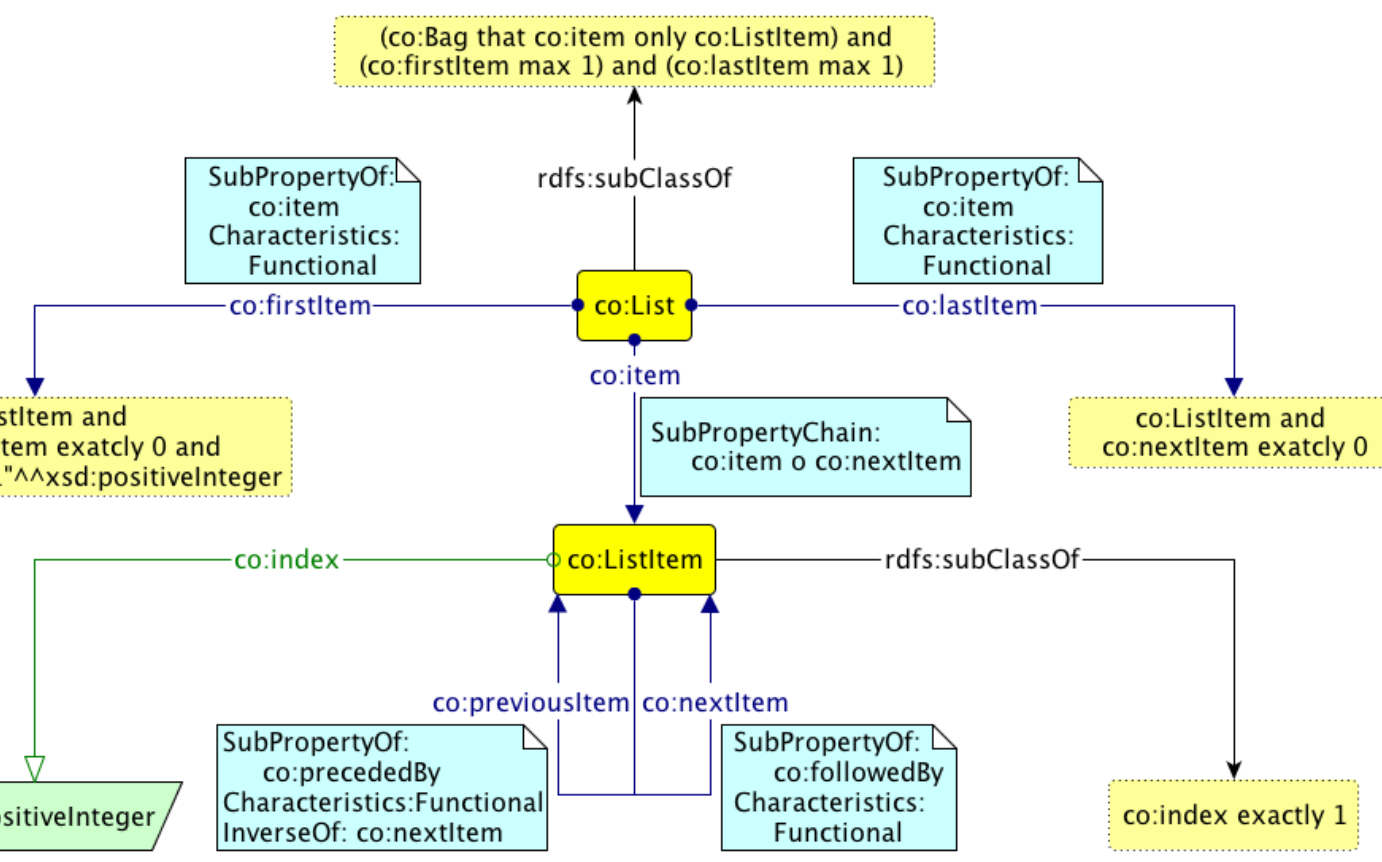

Figure 4. Diagram summarising the class List and the related class ListItem and properties. 